\title{
Wheat homologs of yeast ATG6 function in autophagy and are implicated in powdery mildew immunity
}

\author{
Jieyu Yue ${ }^{\dagger}$, Hong Sun ${ }^{\dagger}$, Wei Zhang, Dan Pei, Yang He and Huazhong Wang*
}

\begin{abstract}
Background: Autophagy-related ATG6 proteins are pleiotropic proteins functioning in autophagy and the phosphatidylinositol 3-phosphate-signaling pathways. Arabidopsis ATG6 regulates normal plant growth, pollen development and germination, and plant responses to biotic/abiotic stresses. However, the ATG6 functions in wheat (Triticum aestivum L.), an important food crop, are lacking.
\end{abstract}

Results: We identified three members, TaATG6a- $6 c$, of the ATG6 family from common wheat. TaATG $6 a, 6 b$ and $6 c$ were localized on homeologous chromosomes 3DL, 3BL and $3 \mathrm{AL}$, respectively, of the allo-hexaploid wheat genome, and evidence was provided for their essential role in autophagy. The TaATG6a-GFP fusion protein was found in punctate pre-autophagosomal structures. The expression of each TaATG6 gene restored the accumulation of autophagic bodies in atg6-mutant yeast. Additionally, TaATG6 knockdown plants showed impaired constitutive and pathogen-induced autophagy and growth abnormalities under normal conditions. We also examined the expression patterns of wheat ATG6s for clues to their physiological roles, and found that their expression was induced by the fungus Blumeria graminis f. sp. tritici (Bgt), the causal agent of powdery mildew, and by abiotic stress factors. A role for TaATG6s in wheat immunity to powdery mildew was further implied when knockdowns of TaATG6s weakly compromised the broad-spectrum powdery mildew resistance gene Pm21-triggered resistance response and, conversely and significantly, enhanced the basal resistance of susceptible plants. In addition, leaf cell death was sometimes induced by growth-retarded small Bgt mycelia on susceptible TaATG6 knockdown plants after a long period of interaction. Thus, we provide an important extension of the previous characterization of plant ATG6 genes in wheat, and observed a role for autophagy genes in wheat immune responses to fungal pathogens.

Conclusions: Three wheat ATG6s were identified and shown to be essential for autophagy biogenesis. Wheat ATG6s are implicated in immunity to powdery mildew, playing a weak, positive role in the Pm21-triggered resistance response and a negative role in the basal resistance of susceptible plants.

Keywords: Autophagy, ATG6, Common wheat (Triticum aestivum L.), Powdery mildew

\section{Background}

Unlike animals, plants are sessile and must overcome or endure variable, sometimes severe environmental conditions. For example, plants have evolved two main defense mechanisms, pathogen-associated molecular pattern (PAMP)-triggered immunity (PTI) and effectertriggered immunity (ETI), to combat pathogen infections [1,2]. ETI is a much stronger reaction than PTI

\footnotetext{
*Correspondence: skywhz@mail.tjnu.edu.cn

${ }^{\dagger}$ Equal contributors

Tianjin Key Laboratory of Animal and Plant Resistance, School of Life Sciences, Tianjin Normal University, Tianjin 300387, China
}

and is often accompanied by a localized hypersensitive response (HR), a type of programmed cell death (PCD), to capture the pathogen at the site of infection. Autophagy is an evolutionarily conserved, eukaryotic process by which organelles and cytosolic macromolecules are consumed in lysosomes (vacuoles in yeast and plants) for nutrient recycling [3-5]. Recent evidence from autophagy-defective Arabidopsis plants suggests an important role for autophagy in regulating plant immune responses [6,7]. This role, however, remains unclear since both positive and negative effects of autophagy on plant immunity have been described in 
different Arabidopsis-pathogen systems as well as in the same Arabidopsis-pathogen system [8,9]. Likewise, both pro-survival and pro-death roles of autophagy in the regulation of HR-PCD or pathogen-induced cell death have been reported [8-10]. Therefore, the mechanisms behind the relationship between autophagy and plant immunity need to be elucidated in detail when considering plant species other than Arabidopsis and more plant-pathogen systems.

During autophagy, double-membrane structures called autophagosomes facilitate the transport of cellular cargos into lysosomes for degradation [3-5]. To date, more than 30 autophagy-related proteins (ATGs) functioning in autophagy induction and regulation, the initiation and biogenesis of autophagosomes, and the fusion of autophagosomes with lysosomes have been identified in yeast and some orthologs identified in animals and plants [3-5]. In yeast, a Class III phosphatidylinositol 3-kinase (PI3K) complex resides in the pre-autophagosomal structure/ phagophore assembly site (PAS) where it catalyzes phosphatidylinositol 3-phosphate (PI3P) synthesis and recruits PI3P-binding proteins, especially the ATG18-ATG2 complex, for the initiation of autophagic membranes [11-13]. Yeast ATG6/vacuolar protein sorting 30 (VPS30), the ortholog of mammalian Beclin 1, is the core component of the PI3K complex along with VPS34, VPS15 and ATG14 $[14,15]$ and is essential for auophagy. Plant homologs of yeast ATG6 play similar essential roles in autophagy, for ATG6-deficient plants produce a reduced number of autolysosomes under autophagy-inducing conditions $[16,17]$, and the expression of plant ATG6 restores autophagy in atg6-mutant yeast [18].

ATG6-deficient plants, like mutants of other atg genes, show an enhanced sensitivity to nutrient starvation and other stress factors $[17,19-22]$. This enhanced sensitivity may result from impaired autophagy because autophagy is responsible for nutrient recycling and distribution under severe environmental conditions. However, the ATG6-associated PI3K complex is not autophagy-specific. Its phospholipid product, PI3P, is a well-known second messenger involved in receptor signaling and protein sorting-related vesicle trafficking events [23,24]. Homozygous plant mutants of atg6, vps34 or vps 15 cannot be obtained because of the requirement of PI3K in pollen development, germination and pollen tube growth [25-27]. Such effects may be attributed to the PI3P-, or its derivative $\mathrm{PI}(3,5) \mathrm{P} 2-$, signaling pathways rather than to autophagy because homozygous mutants of the autophagyspecific atg genes can be routinely obtained by selfing heterozygous individuals. ATG6-deficient plants also show more severe growth abnormalities under normal conditions than mutants of the autophagy-specific atg genes, although they have similarly reduced autophagy levels $[17,22,25,28]$. ATG6, like autophagy-specific factors, is implicated in plant immune responses $[16,17]$. Yet the pleiotropy of ATG6 makes it difficult to interpret whether autophagy, the PI3P-signaling pathways, or their interplay underlines the roles of ATG6 in plant immunity. Well-established roles of ATG6, other than autophagy, also include its involvement in the vacuolar protein sorting pathway of yeast and in tumor suppression through interactions with the anti-apoptotic Bcl-2like proteins in mammals [14,29].

So far, few studies on the mechanisms and physiological functions of autophagy and other ATG6-associated pathways have been reported in crop plants, and fewer still in common wheat (Triticum aestivum L.), which is an important food crop worldwide. Here, we report the identification of three members of the wheat ATG6 family. The conserved function of wheat ATG6s in autophagy was shown using yeast functional complementation, subcellular localization of green fluorescent protein (GFP) fusions and the determination of autophagy levels in ATG6 knockdown plants. A role for ATG6 in the wheat immune response to the causal fungus, Blumeria graminis f. sp. tritici (Bgt), of powdery mildew was established by Bgtinduced ATG6 expression profiles and by altered powdery mildew symptoms on ATG6 knockdown plants.

\section{Methods}

\section{Plant materials and fungal strain}

Wheat used in this study included isogenic lines 92R137/ Yangmai $158^{7}$ and Yangmai 158, and isogenic lines Michigan Amber/Chancellor ${ }^{8}$ and Chancellor. 92R137/ Yangmai $158^{7}$ carries the broad-spectrum powdery mildew resistance gene $P m 21$. Michigan Amber/Chancellor ${ }^{8}$ carries the isolate-specific powdery mildew resistance gene Pm3f. Yangmai 158 and Chancellor are susceptible to powdery mildew.

The prevalent Chinese Bgt isolate E09, which is avirulent to Pm21 and Pm3f, was maintained on seedlings of the susceptible wheat cultivar Sumai 3 under sporeproof conditions.

\section{Plant growth conditions and treatments}

All wheat plants were cultured hydroponically in onefifth strength Hoagland's solution under normal conditions of $22^{\circ} \mathrm{C} / 18^{\circ} \mathrm{C}$ (day/night) and a photoperiod of $16 \mathrm{~h}$ light $/ 8 \mathrm{~h}$ dark in controlled climate chambers. Two-leaf stage seedlings were subjected to different treatments. Pathogen inoculation was conducted by vigorously shaking off fresh Bgt conidia from diseased Sumai 3 plants onto experimental seedlings. For abiotic stress treatments, seedlings were transferred to the same one-fifth strength Hoagland's solution containing $200 \mathrm{mM} \mathrm{NaCl}$ (high salinity) or 16\% PEG6000 (drought). Additional abiotic stress treatments were nitrogen deprivation, and low temperature combined with darkness, in which 
seedlings were transferred to a $4^{\circ} \mathrm{C}$ refrigerator and kept in the dark. Leaves harvested at defined time points were frozen with liquid nitrogen and then stored at $-80^{\circ} \mathrm{C}$. These leaf samples were used for gene isolation and expression analyses.

\section{Isolation of wheat ATG6 genes and expression analyses} Total RNA was prepared from each harvested leaf sample using TRIzol (Invitrogen, Life Technologies, CA, USA) and subjected to RNase-free DNase I digestion and purification. First-strand cDNA was synthesized using oligo(dT) primers and the Quantscript RT Kit (Tiangen, Beijing, China).

The protein sequence of Arabidopsis ATG6 (At3g61710) was used as the input to search for wheat expressed sequence tags (ESTs) in the dbEST division of GenBank by TBLASTN. Both 5'- and 3'-terminal ESTs were iteratively used as queries to search for new terminal ESTs to cover full-length open reading frames (ORFs). EST contigs were assembled in Vector NTI Advance 11.5, and their sequences were used to design primers (Additional file 2: Table S1) flanking full-length ORFs. ATG6 genes were amplified from first-strand cDNA using the high-fidelity Pfu DNA polymerase. Amplified products were A-tailed and then cloned into the vector pGEM ${ }^{\circ}-\mathrm{T}$ Easy (Promega, Madison, WI, USA) for sequencing. Sequence data were deposited in the GenBank database.

The expression patterns of wheat ATG6s responding to biotic and abiotic treatments were quantified by quantitative real-time PCR (qRT-PCR) with gene-specific primers (Additional file 2: Table S1). qRT-PCR was conducted in a $20 \mu$ l RealMasterMix (SYBR Green) mixture (Tiangen, Beijing, China) containing $1 \mu \mathrm{l}$ first-strand cDNA as the template, and the reaction was performed on an IQ5 (BioRad, Hercules, CA, USA). Each cDNA sample was analyzed in triplicate. Relative expression levels were calculated using the $2^{-\Delta \Delta C T}$ method [30] with the amplicon of the wheat $\beta$-tubulin gene as an internal control.

\section{Chromosomal localization of wheat ATG6s}

Wheat nullisomic-tetrasomic lines (NTLs) were used to map ATG6 genes on specific chromosomes. DNA of each line was isolated and used as the template for PCR amplification with gene-specific primers (Additional file 2: Table S1). Amplified products were analyzed by $12 \%$ polyacrylamide gel electrophoresis. Chromosome localization was inferred based on the absence of amplified fragments from lines lacking specific chromosomes.

\section{Complementation test of yeast atg6 mutant}

The ORF of each wheat ATG6 cDNA was amplified as a NotI- NotI fragment using the primers listed in Additional file 2: Table S1. Amplified products were digested and cloned into the yeast expression vector pFL61, in which the constitutive expression of the inserted gene was driven by the phosphoglycinecerate kinase (PGK) gene promoter. Constructed vectors were sequenced to confirm the correct cDNA insertion direction. Wild-type yeast strain BY4741 and a mutant strain, atg6 (BY4741, atg64::kanMX MATa his3 31 leu $2 \Delta 0$ met $15 \Delta 0$ ura3 $\Delta 0$ ), were purchased from Open Biosystems (Thermo Scientific, Wilmington, DE, USA). Each constructed vector was introduced into yeast atg6 cells according to the LiAc/SS-DNA/PEG TRAFO protocol [31]. Positive transformants were screened on SC-U medium. The yeast autophagy complementation test was performed as previously described [18]. The accumulation of autophagic bodies in vacuoles was observed under a differential interference contrast microscope (DM5000B, Leica).

\section{Subcellular localization of wheat ATG6s}

The ORF of each wheat ATG6 cDNA lacking a stop codon was amplified as an XhoI-SpeI fragment using primers listed in Additional file 2: Table S1. Amplified products were digested, and ligated upstream and in-frame with the ORF of GFP in the vector pA7-GFP, in which the GFP fusion construct's expression was driven by two tandem $35 \mathrm{~S}$ promoters. Prepared plasmids were introduced into onion epidermal cells by particle bombardment with PDS1000/ He (BioRad, Hercules, CA, USA). GFP fluorescence was visualized by epifluorescence microscopy (DM5000B, Leica) $24 \mathrm{~h}$ after bombardment.

\section{Virus-induced gene silencing (VIGS)}

A barley stripe mosaic virus (BSMV)-based VIGS method $[32,33]$ was used to create gene knockdown plants. To maximize knockdown efficiency, three $\mathrm{BSMV}_{\gamma}$ vectors, each containing a 200-241 bp fragment from the upstream, middle, or downstream region of the TaATG6a coding sequence, were constructed, and in vitro transcribed $\mathrm{RNA}_{\gamma}$ molecules from these vectors were premixed for virus infection. To circumvent the potential functional redundancy among paralogs, we intended to produce simultaneous knockdowns of wheat ATG6 members by choosing fragments from TaATG6a for the construction of $\mathrm{BSMV}_{\gamma}$ vectors with 94.1-99.5\% sequence identities to the corresponding fragments of TaATG6b and $6 c$ and multiple nucleotide stretches longer than $23 \mathrm{bp}$ that are $100 \%$ identical among the three ATG6 members. The three fragments were prepared by PCR using the primers containing NheI sites listed in Additional file 2: Table S1. Each amplified fragment was digested by NheI and then inserted in the reverse orientation into the

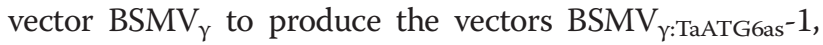

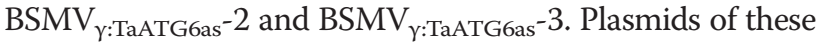
three $B S M V \gamma$ derivatives, as well as $B \mathrm{BMV}_{\gamma: G F P}$ and $\mathrm{BSMV}_{\alpha}$, were linearized by $M l u \mathrm{I}$, and $\mathrm{BSMV}_{\beta}$ was linearized by SpeI. Linearized vectors were in vitro transcribed to 
produce 5 -capped infectious BSMV RNA molecules using the RiboMAX Large Scale RNA Production-T7 kit (Promega, Madison, WI, USA) with the addition of a cap analog (Promega, Madison, WI, USA) in the transcription mixture. RNA R:TaATG6as $-1, \mathrm{RNA}_{\gamma: \text { TaATG6as }}{ }^{2}$, and $\mathrm{RNA}_{\gamma: \text { TaATG6as }}-3$ were premixed in a 1:1:1 ratio to produce $\mathrm{RNA}_{\gamma: \text { TaATG6as. }}$.

The second fully expanded leaves of wheat seedlings at the two-leaf stage were mechanically infected with a 1:1:1 mixture of $\mathrm{RNA}_{\alpha}, \mathrm{RNA}_{\beta}$ and RNA $\mathrm{R}_{\gamma: \text { TaATG6as }}$ (BSMV:ATG6 treatment) in $1 \times$ GKP buffer [33]. Seedlings infected with a 1:1:1 mixture of $\mathrm{RNA}_{\alpha}, \mathrm{RNA}_{\beta}$ and $\mathrm{RNA}_{\gamma: \mathrm{GFP}}$ (BSMV: GFP treatment) in $1 \times$ GKP buffer were used as controls. Three replications of the VIGS experiment were conducted on the two isogenic lines of 92R137/Yangmai $158^{7}$ and Yangmai 158. In each replication, a total of 80 seedlings per line were used for each of the BSMV:ATG6 and BSMV:GFP treatments. The BSMV-treated plants were maintained on one-fifth strength Hoagland's solution under normal conditions. The success of the virus infection was judged by visual observations of mild chlorotic mosaic symptoms on the third and upper leaves of BSMV-treated plants. To confirm the knockdown efficiency, eight plants with virus symptoms were randomly selected from each of the two BSMV treatments, and their transcript levels of TaATG6s in the fourth leaves were determined using qRT-PCR as described above with a primer pair (Additional file 2: Table S1) designed to conserve regions in the three wheat $A T G 6 s$.

\section{Determination of autophagy level by LysoTracker Red staining}

BSMV-treated plants at the four-leaf stage were inoculated with Bgt conidia as described above. The fourth leaves were excised at 0 and 7 days after inoculation (dai) with Bgt, infiltrated with $100 \mu \mathrm{M}$ E-64d (Sigma, St. Louis, MO, USA) and then stained with $2 \mu \mathrm{M}$ LysoTracker Red DND-99 (Invitrogen, Life Technologies, CA, USA) according to the procedure described previously [16]. Fluorescence signals were observed under a confocal microscope (ECLIPSE 90i, Nikon) using a 543$\mathrm{nm}$ excitation laser and 565 to $615-\mathrm{nm}$ band pass.

\section{Chlorophyll content determination}

The fifth leaves of BSMV-treated plants at the five-leaf stage were excised, weighed and ground in $1 \mathrm{ml}$ of $80 \%$ $(\mathrm{v} / \mathrm{v})$ acetone. The chlorophyll content was determined spectrophotometrically according to Patel and DineshKumar [17].

\section{Leaf senescence assay}

Green sections were excised from the fourth leaves of BSMV-treated plants at the four-leaf stage and floated on deionized water at room temperature in the dark.
Leaf sections were photographed every day after dark incubation. The experiment was repeated in triplicate using leaves of three or more plants in each experiment.

\section{Evaluation of powdery mildew resistance}

To exclude the influence of early senescence/chlorosis on Bgt infections, only those BSMV:ATG6-treated plants without obvious symptoms of early senescence and chlorosis were selected for resistance evaluation. BSMV-treated plants at the six-leaf stage were inoculated with Bgt conidia as described above. For BSMV-treated Yangmai 158 plants, leaf discs with visible mildew colonies were gently harvested from different regions of the fourth leaves at 5 dai, immersed in $0.01 \%$ glycerol and subjected to an ultrasonic treatment for $1 \mathrm{~min}$ to disperse the conidia. The conidial production per mildew colony was counted by microscopic observation and expressed as the mean \pm SD of at least 50 mildew colonies from five plants.

Fungal structures were stained by trypan blue [34] and visualized by light microscopy. For BSMV-treated 92R137/Yangmai $158^{7}(P m 21)$ plants, the number of successfully penetrating conidium with secondary hyphae on the fourth leaves was counted at 7 dai and expressed as a percentage of at least 100 interaction sites on individual plants. The number of secondary hypha associated with each successfully penetrating conidium was also counted and expressed as the mean \pm SD of the observed successfully penetrating conidia on all of the investigated plants. For BSMV-treated Yangmai 158 plants, the number of mycelium with mature chains of conidia on the fourth leaves was counted at 5 dai and expressed as a percentage of at least 100 mycelia on the leaves of five plants.

\section{Statistical analysis}

All experiments were repeated three times with similar results. Quantitative data were statistically analyzed using Student's $t$ test $(\mathrm{P}<0.05)$ using the IBM SPSS19.0 software package.

\section{Results}

\section{Isolation of ATG6 homologs from wheat}

Using the sequence of Arabidopsis ATG6 as a query, 44 putative wheat ATG6-encoding ESTs were mined from the dbEST division of GenBank and assembled into contigs. Primers covering the full-length ORFs of these contigs were designed and used for gene isolation by PCR from a leaf cDNA pool of the 92R137/Yangmai $158^{7}$ (Pm21) plants induced by Bgt for $48 \mathrm{~h}$. Three genes, which encoded a typical ATG6 domain (Pfam PF04111) (Additional file 1: Figure S1A), were identified as ATG6 homologs in wheat and designated as TaATG6a [GenBank: U49845], $6 b$ [GenBank: U49845] and $6 c$ [GenBank: U49845]. The ORF nucleotide sequences of 
TaATG6a, $6 b$ and $6 c$ share identities of $96.1-98.4 \%$ and encode peptide sequences of 504, 500, and 503 amino acids, respectively, with $98-99 \%$ similarity. In the eukaryotic ATG6 phylogenetic tree (Additional file 1: Figure S1B), plant ATG6s are separately grouped into monocot and dicot clades. TaATG6a-6c showed $90-93 \%$ sequence similarities to OsATG6a-6c from rice and 75\% to AtATG6 from Arabidopsis. The ATG6 domain region is more conserved across all plant ATG6s (80-89\% similarity), whereas the $\mathrm{N}$ - and $\mathrm{C}$-terminal flanking sequences show divergence between the monocot and dicot ATG6s (Additional file 1: Figure S1A).

Two methods, gene-specific PCR amplification of wheat NTL DNAs and BLAST searches against the mapped wheat genomic sequences (genome survey sequences and the completed reference sequence of chromosome 3B) [35], were performed to determine the chromosomal localizations of wheat ATG6 members. TaATG6b and $6 c$ were localized on chromosomes $3 \mathrm{BL}$ and $3 \mathrm{AL}$, respectively, by both methods (Figure 1). TaATG6a was localized on chromosome 3DL based only on the results of BLAST searches.

We also obtained the respective genomic sequences of TaATG6 $a, 6 b$ and $6 c$ by BLAST searches against wheat genomic sequences. Similar nine-intron structures of the TaATG6a-6c ORF sequences were revealed when comparing genomic sequences with the corresponding cDNA sequences (Additional file 1: Figure S2). However, TaATG6b has a much longer intron 3 than TaATG6a and $6 c$. We compared the three genomic sequences and found a 900-bp insertion only in the intron 3 of TaATG6b, which is bordered by 19-bp direct short sequence repeats (TAGACTTAAATCATACTCC) (Additional file 1: Figure S2). As it encodes a truncated protein similar to RNAdirected DNA polymerases and polyproteins of mobile elements and because no long terminal repeats (LTR) were found around it, this inserted sequence most likely resulted from a transposition event of a non-LTR retrotransposon. A CpG island of $999 \mathrm{bp}$, covering the start codon, was detected in the TaATG6b genomic sequence, which has a $60.1 \% \mathrm{G}+\mathrm{C}$ content and a ratio of 1.07 of the observed versus expected presence of CpG dinucleotides (Figure 2A). This CpG island exists in other monocot $A T G 6 \mathrm{~s}$ as well, including OsATG6b from rice, but not in dicot ATG6s, such as AtATG6 from Arabidopsis (Figure 2A). Within the CpG island, there is a short GCrich stretch just downstream of the start codon, and its length varies among paralogs as well as among homologs (Figure 2B). Only insertions or deletions that involve multiples of three bases are retained in the GC-rich stretch of monocot ATG6s because they do not result in translational frame shifts. Amino acids encoded in the GC-rich stretch are predominantly glycines and alanines.

\section{Wheat ATG6s function in autophagy}

Human and Arabidopsis ATG6 can rescue the autophagy activity of atg6-mutant yeast $[16,18,36]$. To determine if wheat ATG6s are functional homologs of yeast ATG6, yeast expression vectors were constructed for each TaATG6 and transformed into atg6-mutant yeast cells. Autophagic bodies in vacuoles were monitored as an indicator of autophagy activity. After $5 \mathrm{~h}$ of nutrient starvation and treatment with the protease inhibitor phenylmethylsulfonyl fluoride, the wild-type yeast cells accumulated numerous autophagic bodies in vacuoles, which were rarely observed in the atg6-mutant yeast cells (Figure 3A). Cells of atg6-mutant yeast transformed with TaATG6a, $6 b$ or $6 c$ partially restored the accumulation of autophagic bodies (Figure 3A).

Punctate structures of ATG6-GFP fluorescence, considered as PAS for autophagosome initiation, have been observed in cells of yeast and Arabidopsis [18,37]. Here, GFP fusion constructs of wheat ATG6s were introduced into onion epidermal cells by particle bombardment. Punctate florescence signals were clearly observed in the cytoplasm of cells expressing TaATG6a-GFP and seemed not to occur in the central vacuole (Figure 3B). Fluorescent spots of TaATG6a-GFP were sometimes clustered at cytoplasmic and perinuclear membrane-like structures resembling the trans-Golgi network and endoplasmic reticulum (Figure 3C). Localizations of ATG6s to endosomes, the trans-Golgi network, endoplasmic reticulum,

\section{$\mathrm{N}$, nullisomic; $\mathrm{T}$, tetrasomic}

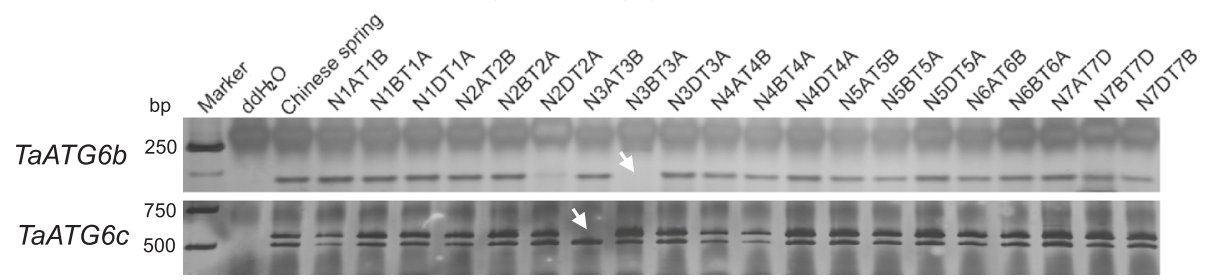

Figure 1 Chromosomal localization of wheat autophagy-related ATG6 genes. DNA of each wheat nullisomic-tetrasomic line was used as a template for PCR amplification with gene-specific primers. Amplified products were analyzed on $12 \%$ polyacrylamide gels. Chromosome localization was inferred based on the absence of amplified fragments (arrows) in lines lacking the specific chromosomes. 


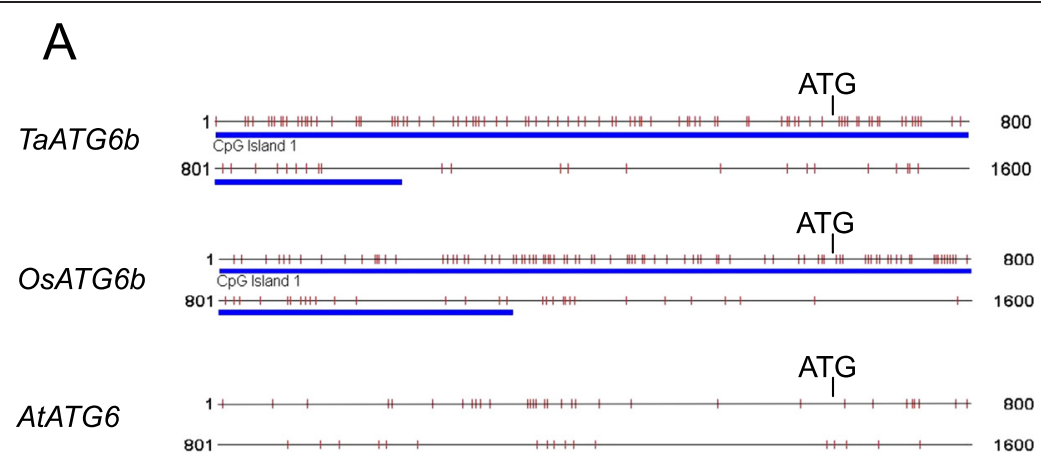

B

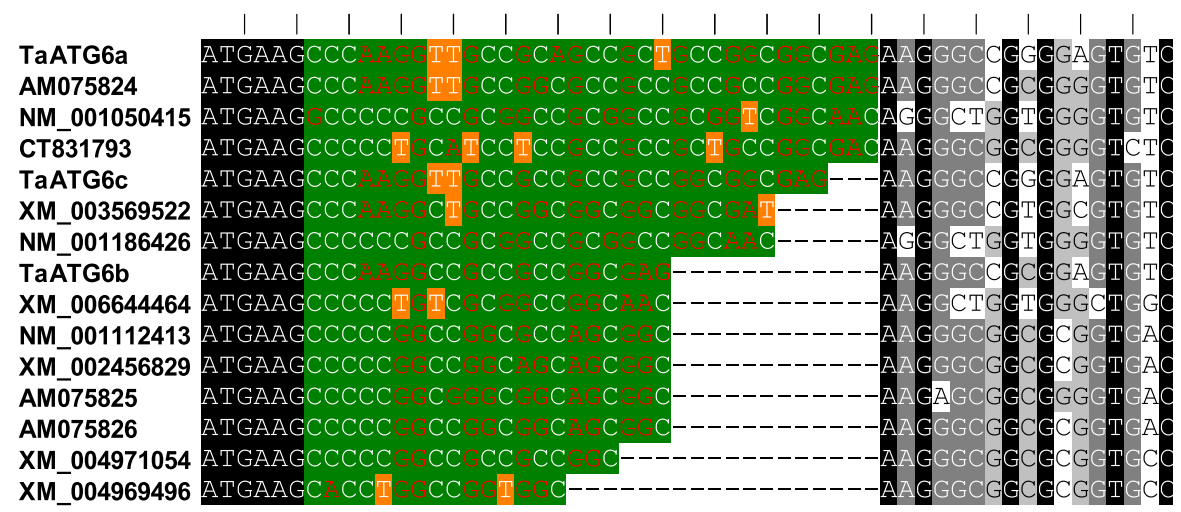

Figure $\mathbf{2}$ Characterization of wheat autophagy-related ATG6 sequences. (A) The genomic sequences of monocot ATG6s contain a CpG island covering the start codon. The CpG island was predicted using the web tool CpG Island Searcher (http://cpgislands.usc.edu/) with a CpG island standard of longer than $500 \mathrm{bp}$ in length, a minimum $\mathrm{G}+\mathrm{C}$ content of $55 \%$ and a minimum 0.65 ratio of the observed versus the expected presence of $\mathrm{CpG}$ dinucleotides. Gray, horizontal lines represent the 1600-bp start codon-covering genomic sequences of TaATG6b from wheat, OsATG6b (Os03g0644000) from rice and AtATG6 (At3g61710) from Arabidopsis. Short vertical red lines represent CpG dinucleotides. Blue horizontal thick lines represent the ranges of predicted CpG islands. (B) Short GC-rich stretch just downstream of the start codon in monocot ATG6 sequences. Sequences shown are the 5'-terminal cDNA sequences beginning with the start codons of monocot ATG6s, including TaATG6a, $6 b$ and 6c from wheat, AM075824 from Hordeum vulgare, NM_001050415, CT831793 and NM_001186426 from rice, XM_003569522 from Brachypodium distachyon, XM_006644464 from Oryza brachyantha, NM_001112413 from Zea mays, XM_002456829 and AM075825 from Sorghum bicolor, AM075826 from Saccharum officinarum, and XM_004971054 and XM_004969496 from Setaria italic. The GC-rich stretches (green background) are not properly aligned but the flanking sequences are aligned. The reading frames from ATG are indicated by the upper vertical lines.

mitochondria, cytoplasm, and nucleus have been reported in yeast and human [37-40]. Unexpectedly, TaATG6band TaATG6c-GFP florescence showed diffused distributions in the cytoplasm and nuclei, which were almost indistinguishable from those in cells expressing GFP alone (Figure 3B). Therefore, additional signals may be required to trigger the translocation of TaATG6b and $6 c$ to PAS for their role in autophagy initiation.

To determine if an ATG6 deficiency can lead to autophagy impairment in wheat, BSMV:ATG6-treated TaATG6 knockdown plants were generated by the VIGS method and their autophagy levels were compared with BSMV: GFP-treated control plants. To obtain the maximum knockdown efficiency, BSMV:ATG6 was designed to contain a mix of three BSMV $\mathrm{RNA}_{\gamma}$ molecules, each containing an inserted antisense fragment corresponding to the upstream, middle, and downstream regions of the TaATG6a coding sequence. Taking into consideration the potential functional redundancy among paralogs in polyploid wheat, BSMV:ATG6 was also designed to knock down all three wheat ATG6 members by inserting three fragments in the $\mathrm{RNA}_{\gamma}$ molecules that are highly conserved (more than $94.1 \%$ identical) among the three genes. As determined by qRT-PCR using a primer pair designed based on identical regions of the three TaATG6s, the total constitutive transcript levels of TaATG6a-6c were greatly reduced in the BSMV:ATG6treated plants compared with the transcript levels in BSMV:GFP-treated control plants (Figure 3D). This high knockdown efficiency was achieved in all eight BSMV:ATG6-treated plants investigated, which confirmed the effectiveness of our VIGS design. We then inoculated BSMV-treated plants with Bgt conidia and determined their constitutive autophagy activity at 0 dai and 

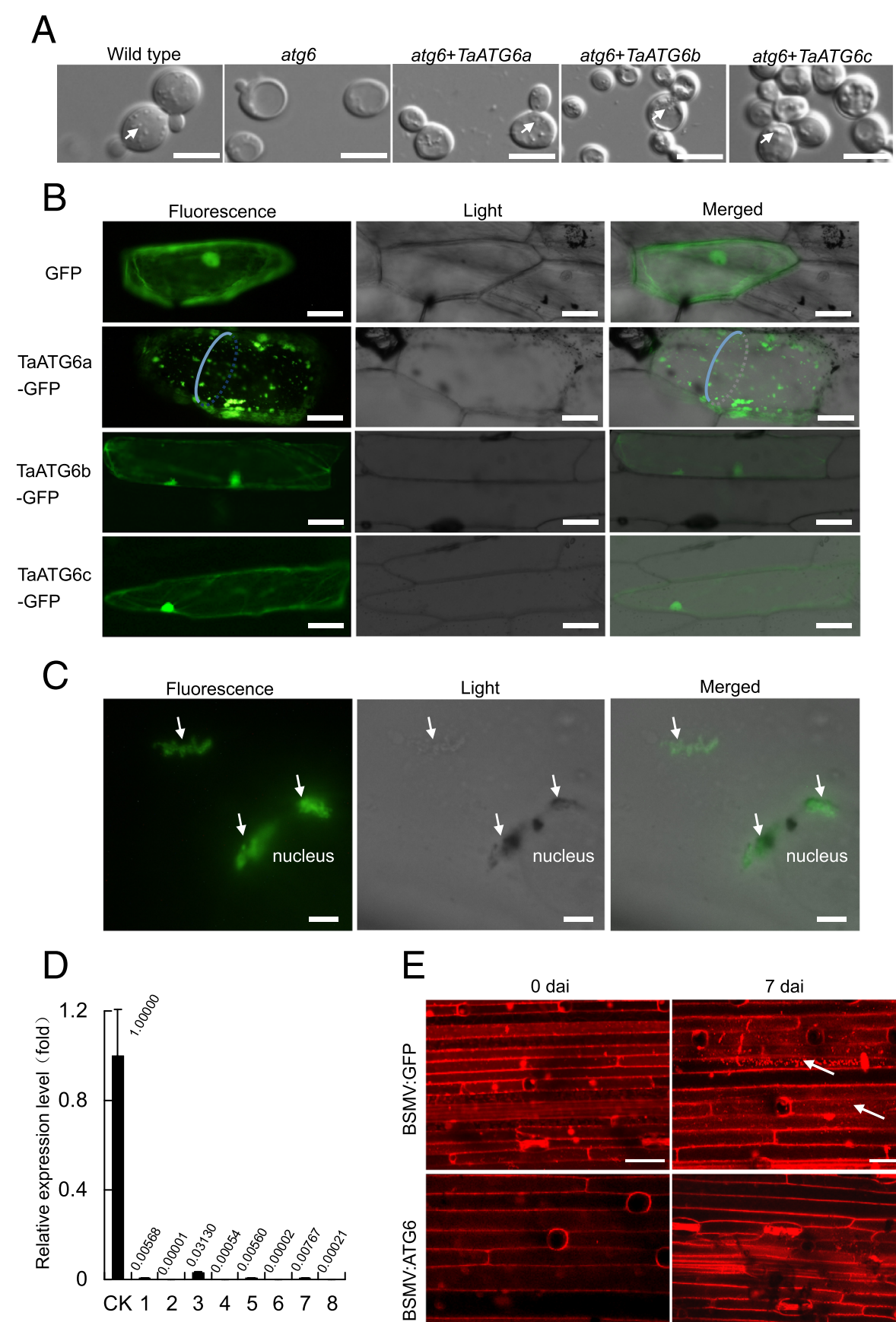

$E$

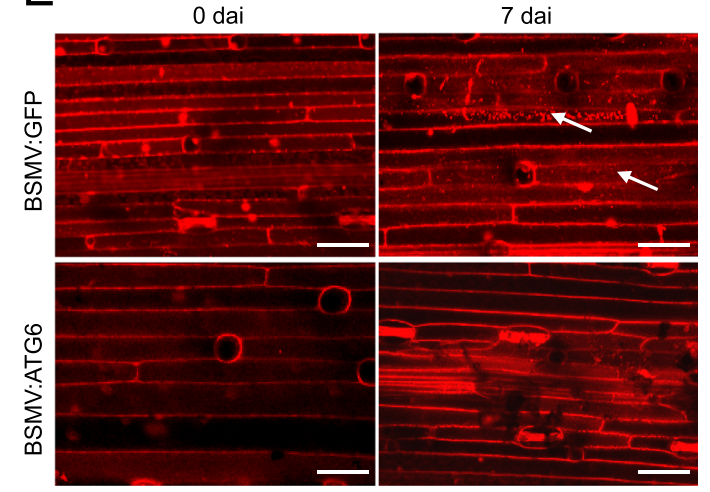

Figure 3 Autophagy-related ATG6 genes function in wheat autophagy. (A) Functional complementation of atg6-mutant yeast cells by wheat ATG6s. The autophagic bodies accumulated within vacuoles are indicated by arrows. Scale bars represent $5 \mu \mathrm{m}$. (B) Subcellular localization of GFP-TaATG6 fusion structures in onion epidermal cells. Scale bars represent $50 \mu \mathrm{m}$. (C) Localization of GFP-TaATG6a in cytoplasmic and perinuclear membrane-like structures in onion epidermal cells. Florescence spots localized in cytoplasmic and perinuclear membrane-like structures are indicated by arrows. Scale bars represent 10 m. (D) Relative transcript levels of wheat ATG6s in leaves of barley stripe mosaic virus (BSMV)-treated plants. The fourth leaves were sampled from BSMV-treated 92R137/Nangmai $158^{7}$ (Pm21) plants under normal conditions and used in the quantification of TaATG6 transcripts by qRT-PCR with a primer pair designed based on identical regions of TaATG6a, $6 b$, and $6 c$. Amplification of the wheat $\beta$-tubulin gene served as an internal control. CK is from BSMV: GFP-treated control plants and Arabic numbers indicate individual BSMV:ATG6-treated plants. (E) Constitutive and Blumeria graminis f. sp. tritici (Bgt)-induced autophagy are impaired in BSMV:ATG6-treated 92R137/Yangmai 158 (Pm21) plants. The fourth leaves were excised from BSMV-treated plants at 0 and 7 days after inoculation (dai) with Bgt conidia, infiltrated with E-64d and then stained with LysoTracker Red. LysoTracker Red-stained punctate autolysosome-like structures are indicated by arrows. The results were reproduced from three independent experiments using three or more plants in each experiment. Scale bars represent $5 \mu \mathrm{m}$. 
Bgt-induced autophagy activity at 7 dai using the LysoTracker Red staining method. LysoTracker Red is a dye that stains acidic organelles, such as lysosomes and endosomes, as well as autophagosomes/autolysosomes (vacuoles and small lytic organelles). Punctate structures labeled by this dye are generally considered indicative of plant autophagy activity [16,41-44]. Before LysoTracker Red staining, leaf samples were pre-infiltrated with the cysteine protease inhibitor E-64d to enhance the accumulation of autophagic bodies inside autolysosomes $[16,45,46]$. For the BSMV:GFP-treated control plants of the resistant line 92R137/Yangmai $158^{7}$ (Pm21), low levels of a LysoTracker Red-stained punctate pattern were observed at 0 dai, and enhanced levels were observed at 7 dai in Bgt-challenged leaf epidermal cells (Figure 3E). These Bgt-enhanced levels implied that the TaATG6s-regulating autophagy process was induced and implicated in the Pm21-triggered immune response to powdery mildew. However, because of the knocking down of TaATG6s, the LysoTracker Red-stained punctate structures were not visible in leaf samples of BSMV:ATG6-treated plants regardless of Bgt challenge (Figure 3E). This result of impaired autophagy in TaATG6 knockdown plants, together with the complementation of yeast autophagy-defective cells with each TaATG6 and the observation of PAS localization of TaATG6a-GFP, demonstrates the essential role of TaATG6s in wheat autophagy.

\section{Wheat ATG6 knockdown plants exhibited growth abnormalities}

BSMV:ATG6-treated plants, when compared with control plants, showed varying degrees of growth defects, including stunted growth and chlorosis under normal conditions (Figure $4 \mathrm{~A}-\mathrm{C}$ ). Some were thin and weak, and a higher percentage of BSMV:ATG6-treated plants shifted into early senescence and accelerated death before the five-leaf stage than of the controls (Figure 4D). To further monitor senescence, green leaf sections from plants at the same developmental stage were excised and kept floating on water in the dark to induce senescence. Leaf sections from BSMV:ATG6-treated plants began senescing within $3 \mathrm{~d}$ post-detachment, as indicated by yellowing (Figure 4E). This was significantly earlier than leaf sections from control plants, which began senescing by $7 \mathrm{~d}$ post-detachment. Thus, TaATG6s are essential for normal plant growth and to prevent premature senescence in wheat.

\section{Expression of wheat ATG6s in response to abiotic stress factors and fungal infection}

Yangmai 158 plants were studied using qRT-PCR to determine the effects of abiotic stress factors on the expression of wheat ATG6s. The expression levels of TaATG6b and $6 c$ were upregulated by high salinity, drought, low temperature/darkness and nitrogen deprivation (Figure 5A). The expression of TaATG6a was also upregulated by high salinity, drought and nitrogen deprivation, but downregulated by low temperature/ darkness (Figure 5A). Among the four investigated abiotic stress factors, wheat ATG6s were most sensitive to the two osmotic stress factors, high salinity and drought, with up to 63-fold (TaATG6a responding to high salinity) and 230 -fold (TaATG6a responding to drought) increases in the accumulation of transcripts. These upregulated expression modes indicate that TaATG6s are closely related to the wheat's responses to abiotic stress factors.

Two powdery mildew resistant lines, 92R137/Yangmai $158^{7}$ (Pm21) and Michigan Amber/Chancellor ${ }^{8}$ (Pm3f), and their respective isogenic susceptible lines were included in the expression analysis, using qRT-PCR, of wheat ATG6s responding to Bgt infection. In the broadspectrum resistance gene $P m 21$ - and the isolate-specific resistance gene $P m 3 f$-triggered resistance responses, the expression of wheat ATG6s was upregulated by Bgt, producing bimodal profiles over a time interval of 0 to $36 \mathrm{~h}$ after inoculation (hai) with Bgt (Figure 5B). The two peaks of transcript accumulation occurred at 10 and 24 hai. Upon Bgt challenge, Pm21 activated the three ATG6s to about the same extent, while Pm3f activated TaATG6a to a greater extent than TaATG6b and $6 c$. In the susceptible response of Yangmai 158 to Bgt, however, a single instance of upregulated expression within 16 to 24 hai was detected for the three ATG6s (Figure 5B). This corresponded to, but was higher than, the second instance of upregulated expression detected in the two resistance responses. In the susceptible response of Chancellor to Bgt, only one member, TaATG6a, showed a Bgt-induced expression profile (Figure 5B). These Bgt-upregulated expression patterns implied that TaATG6s are involved in the wheat immune response to powdery mildew.

\section{Wheat ATG6s play a weak, positive role in the Pm21- triggered resistance response to $\mathrm{Bgt}$}

We used TaATG6 knockdown plants to test if TaATG6s have any effect on Bgt infection. To exclude the influence of early senescence/chlorosis on Bgt infection, only those knockdown plants that did not show obvious early senescence and chlorosis were selected for resistance evaluation. BSMV-treated plants were heavily inoculated with $B g t$ conidia, and disease symptoms were determined when mildew colonies were apparently visible on susceptible plant leaves. The knocking down of TaATG6s did not result in visible mildew colonies on leaves of BSMV: ATG6-treated 92R137/Yangmai $158^{7}$ (Pm21) plants by 7 dai, which seemed to retain a resistant phenotype similar to BSMV:GFP-treated control plants. We then stained leaf samples with trypan blue and, under a microscope, counted the number of successfully penetrating $B g t$ 
A

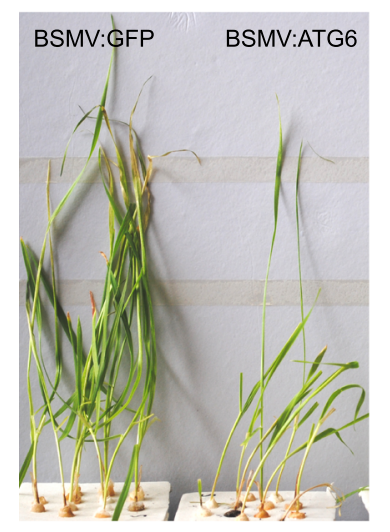

D

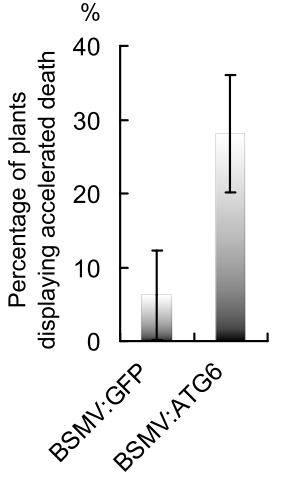

B

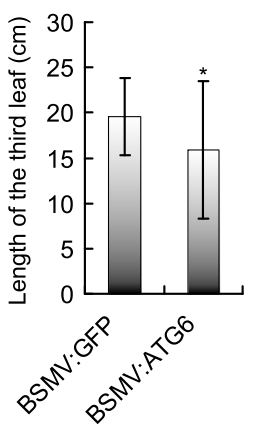

E

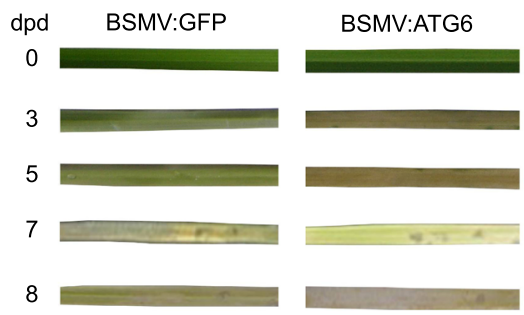

Figure 4 Wheat autophagy-related ATG6 knockdown plants display growth abnormalities. All barley stripe mosaic virus (BSMV)-treated plants were cultured hydroponically in one-fifth strength Hoagland's solution and kept under normal conditions of $22^{\circ} \mathrm{C} / 18^{\circ} \mathrm{C}$ (day/night) and a photoperiod of $16 \mathrm{~h}$ light/8 h dark in controlled climate chambers. (A) The growth morphology of BSMV-treated Yangmai 158 plants. Photographs were taken at the three-leaf stage. The second leaves, which had been mechanically infected with BSMV, were generally withered by this stage and thus were excised before photographing. (B) Leaf length of BSMV-treated Yangmai 158 plants. The lengths of the third leaves were measured at the three-leaf stage. Values represent the mean \pm SD (standard deviation) of data measured from 30 individual plants. Asterisks indicate a significant difference ( $P<0.05$, Student's $t$ test) between the BSMV:ATG6-treated plants and BSMV:GFP-treated control plants. (C) Chlorophyll content of BSMV-treated Yangmai 158 plants. The chlorophyll content of the fifth leaves was determined at the five-leaf stage. Values represent the mean \pm SD of data determined from five individual plants. Asterisks indicate a significant difference $(P<0.05)$ between the BSMV:ATG6-treated plants and BSMV:GFPtreated control plants. (D) Percentage of BSMV-treated Yangmai 158 plants displaying accelerated death before the five-leaf stage. Values represent the mean percentage \pm SEM (standard error of the mean) of at least 30 plants. (E) Detached leaves from BSMV:ATG6-treated plants display accelerated dark-induced senescence. Green sections of the fourth leaves were excised from BSMV-treated Yangmai 158 plants at the four-leaf stage and were floated on deionized water at room temperature in the dark. Leaf sections were photographed at the indicated days post-detachment (dpd). The results are reproduced from three independent experiments using the leaves of three or more plants in each experiment

conidium with secondary hyphae. As shown in Figure 6A and $\mathrm{B}$, the percentage of successfully penetrating $B g t$ conidia (penetration frequency) was very low $(0.5 \%)$ on leaves of the highly resistant BSMV:GFP-treated control plants, but significantly increased to $1.7-29.1 \%$ on leaves of different BSMV:ATG6-treated plants. Furthermore, successfully penetrating Bgt conidia on leaves of BSMV: ATG6-treated plants developed an average of $4.16 \pm 2.91$ prolonged secondary hyphae, whereas only one short secondary hypha was associated with successfully penetrating $B g t$ conidia on leaves of BSMV:GFP-treated control plants (Figure 6B). These results indicated that knocking down
TaATG6s resulted in a compromised Bgt resistance on 92R137/Yangmai $158^{7}$ (Pm21) plants. Therefore, we propose that TaATG6s play a positive role in Pm21triggered wheat resistance response to $B g t$ and that this positive role is weak since no large, sporulating mycelia were observed on TaATG6 knockdown plants of 92R137/ Yangmai $158^{7}$ (Pm21) by 7 dai. In ATG6, VPS34, ATG3, or ATG7 knockdown tobacco plants and in ATG6 knockdown or atg5 mutant Arabidopsis plants, avirulent viral or bacterial pathogen-induced HR-PCD was unrestricted and spread into uninfected cells, suggesting that autophagy has a pro-survival function in the pathogen-induced cell 

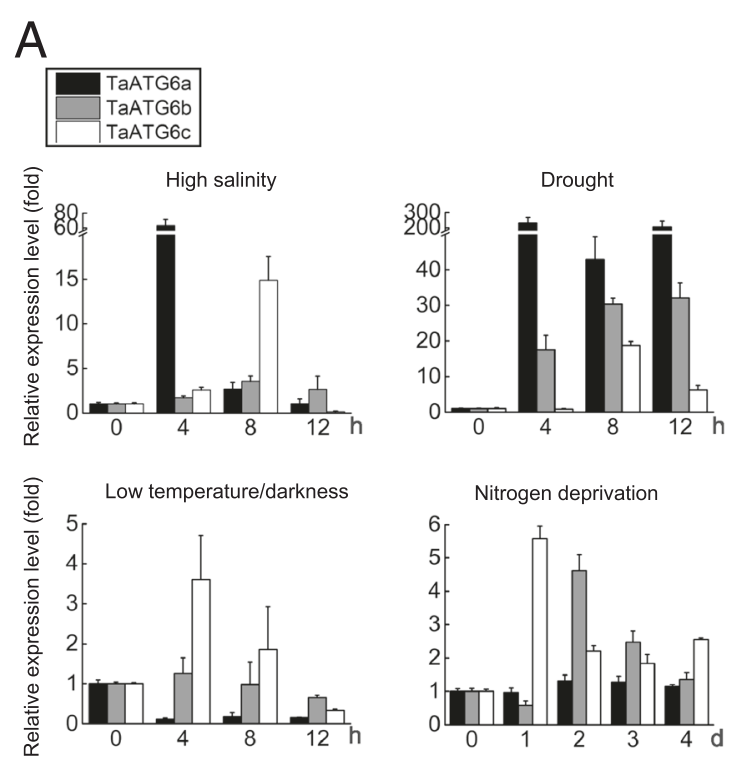

B
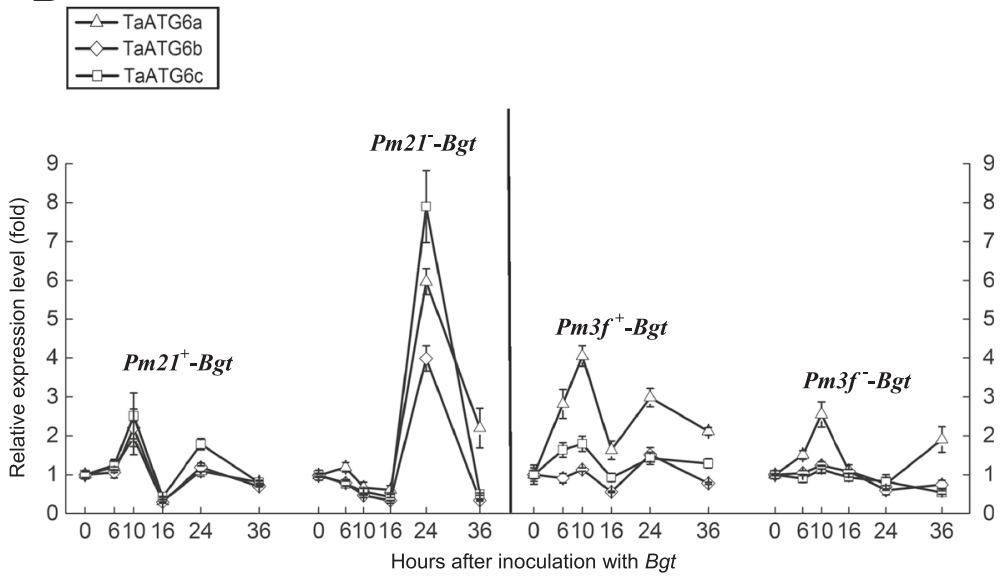

Figure 5 Expression patterns of wheat autophagy-related ATG6 genes. Two-leaf stage seedlings were subjected to different treatments. Samples were collected at the indicated time points after the initiation of treatment. Total RNA was extracted and GRT-PCR was performed with gene-specific primers. The relative expression levels were normalized by the wheat $\beta$-tubulin gene and relative to the control value measured at $0 \mathrm{~h}$. Data represent the mean of three independent experiments \pm SD. (A) Expression of wheat ATG6s in response to abiotic stress factors. Yangmai 158 seedlings were transferred to the one-fifth strength Hoagland's solution containing $200 \mathrm{mM} \mathrm{NaCl}$ (high salinity) or 16\% PEG6000 (drought). Additional seedling treatments were nitrogen deprivation and exposure to $4^{\circ} \mathrm{C}$ in the dark (low temperature/darkness). (B) Expression of wheat ATG6s in response to Blumeria graminis f. sp. tritici (Bgt) infection. Pathogen inoculations were conducted by vigorously shaking fresh Bgt conidia from diseased plants onto seedlings of the isogenic lines 92R137/Yangmai $158^{7}\left(\mathrm{Pm}_{2} 1^{+}\right)$and Yangmai $158\left(\mathrm{Pm} 21^{-}\right)$, and the isogenic lines Michigan Amber/Chancellor ${ }^{8}\left(\mathrm{Pm}^{\mathrm{f}} \mathrm{f}^{+}\right)$and Chancellor $\left(\mathrm{Pm}^{\mathrm{f}} \mathrm{f}^{-}\right)$.

death response $[16,17,44,47]$. In our study, however, such an unrestricted spread of cell death was not induced by the biotrophic fungal pathogen Bgt on TaATG6 knockdown plants of 92R137/Yangmai $158^{7}$ (Pm21).

\section{Wheat ATG6s also play a negative role in the basal} resistance of plants susceptible to Bgt

Although the BSMV:ATG6-treated plants and BSMV: GFP-treated control plants of Yangmai 158 were both colonized by Bgt at 5 dai, the sizes of visible mycelia and their sporulation (conidial production per mildew colony) were significantly reduced on both lower leaves (the fourth leaves, Figure 7A and B) and upper leaves (the sixth leaves, Figure 8A) of BSMV:ATG6-treated plants. Bgt mycelia were also examined under a microscope after trypan blue staining, and a larger portion of small mycelia and a significantly reduced percentage of mycelia with mature chains of conidia were observed on 


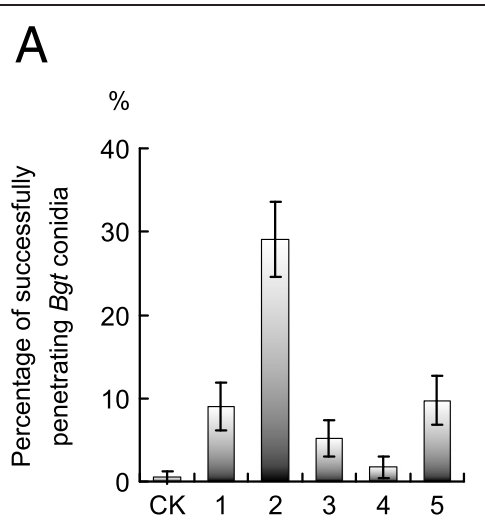

B

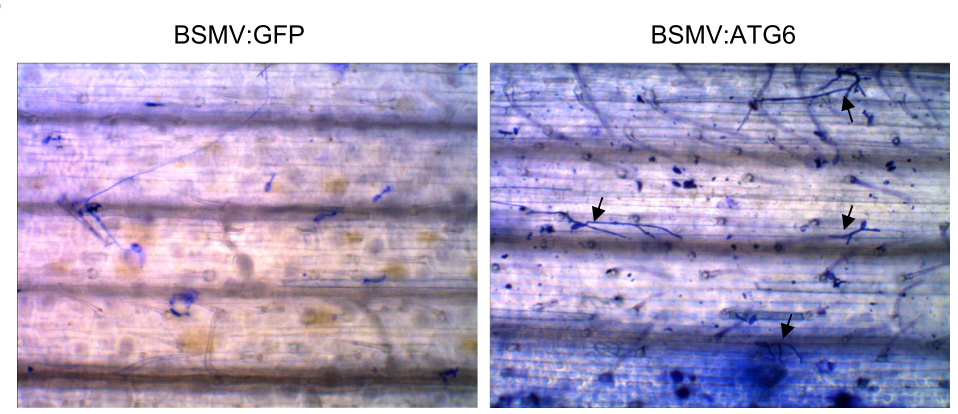

Figure 6 Knocking down wheat autophagy-related ATG6 genes weakly compromises the broad-spectrum resistance gene Pm21-triggered resistance response to Blumeria graminis f. sp. tritici (Bgt). Barley stripe mosaic virus (BSMV)-treated 92R137/Yangmai 158 (Pm21) plants of the six-leaf stage were heavily inoculated with Bgt conidia. Fungal structures were stained by trypan blue and visualized by microscopy. (A) Bgt penetration frequency. The number of successfully penetrating conidium with secondary hyphae on the fourth leaves was counted at 7 days after inoculation (dai) with Bgt conidia. Values represent the mean percentage of successfully penetrating Bgt conidia \pm SEM calculated from at least 100 interaction sites. CK is from BSMV:GFP-treated 92R137/Nangmai 158 (Pm21) control plants. Arabic numbers indicate individual BSMV:ATG6-treated 92R137/Nangmai 158 (Pm21) plants. (B) Representative images of Bgt infection on the fourth leaves of BSMV-treated 92R137/Nangmai 158 (Pm21) plants at 7 dai. Successfully penetrating conidia with secondary hyphae are indicated by arrows.

leaves of BSMV:ATG6-treated plants at 5 dai (Figure 7C). Clearly, a significantly enhanced resistance should be responsible for the retarded mycelial spread and sporulation on TaATG6 knockdown plants. We thus proposed that TaATG6s play negative roles in the basal resistance of the susceptible line Yangmai 158 to powdery mildew. Moreover, growth-retarded small mycelia, after a prolonged infection time of 9-10 dai, induced lesions and chlorosis around lesions on the middle-to-tip leaf regions of BSMV:ATG6-treated Yangmai 158 plants (Figure 8A, left panel of 8B). The exhibition of enhanced resistance preceded the occurrence of leaf lesions and not all small mycelia were finally associated with leaf cell death (Figure 8B), although leaf cell death may further impair the biotrophic lifestyle of Bgt.

\section{Discussion}

As a key component of the Class III PI3K kinase complex, ATG6 is essential for autophagy and the PI3P-signaling pathways. Previously, plant ATG6 orthologs had been identified in Nicotiana benthamiana, Arabidopsis and rice, and their involvement in autophagy had been demonstrated $[16,17,48]$. Here, we characterized three wheat ATG6 genes, TaATG6a, $6 b$ and $6 c$, which were localized on the homeologous chromosomes 3DL, 3BL and 3AL, respectively, of the allo-hexaploid wheat genome (AABBDD). Heterologous expression of TaATG6a, $6 b$, or $6 c$ can restore autophagy in atg6-mutant yeast cells. The TaATG6a-GFP fusion protein was located in cytoplasmic punctate PAS structures. Constitutive and pathogen-induced autophagy processes were impaired in TaATG6 knockdown plants. Collectively, these results support the essential role of TaATG6s in wheat autophagy biogenesis. Consistent with Arabidopsis plants deficient in the PI3K components ATG6 or VPS34 [17,22,49], TaATG6 knockdown wheat plants exhibit abnormal growth phenotypes, such as dwarfing, chlorosis, and accelerated senescence/death, under normal conditions. However, the role of PI3K on normal plant growth may be beyond autophagy, as mutants of 


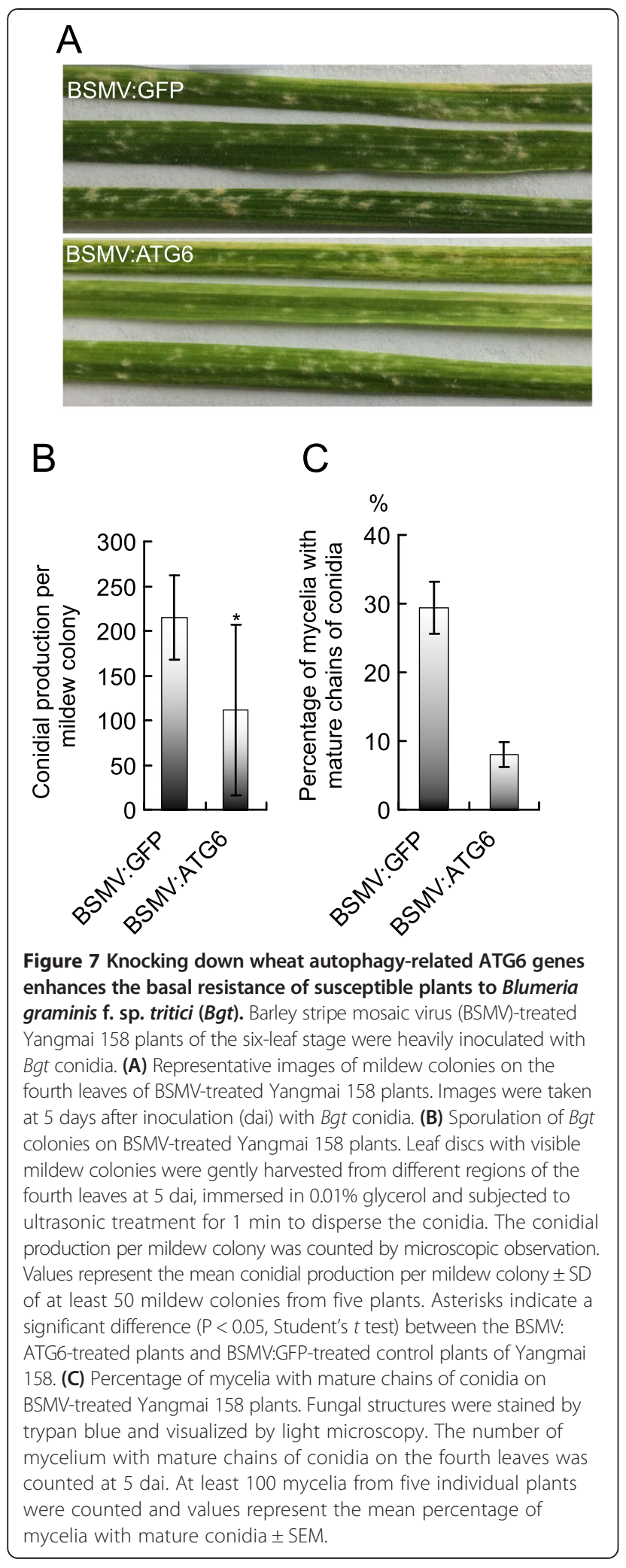

autophagy-specific atg generally do not show such extensive growth abnormalities under normal conditions [19-21,28,50].
Pathogen-induced expression patterns and positive roles of ATG6 in plant innate immunity have been reported in plant pathosystems, such as $N$. benthamianaTMV [16] and Arabidopsis-Pseudomonas syringae [17]. Here, two transcript accumulation events, an early and a late one, of wheat ATG6s were induced in the Pm21and Pm3f-triggered wheat resistance responses to Bgt within 0 to 36 hai. Bgt-induced bimodal expression profiles have also been characterized for other powdery mildew resistance-related genes in wheat [51,52]. Two pathogen-induced oxygen burst processes reported previously also match the two time points [53]. The early wheat ATG6 transcript accumulation event occurred within 0 to 10 hai, which is when Bgt appressorium germ tubes contact and attempt to invade wheat epidermal cells. This transcript accumulation may be pivotal for the Pm21-triggered resistance response because it was not detected in the isogenic susceptible plants of Yangmai 158. Although knocking down TaATG6s did not result in a striking change in the Pm21-triggered resistance phenotype, an increased penetration frequency of inoculated Bgt conidia was observed by microscopy on knockdown plants of 92R137/Yangmai $158^{7}$ (Pm21). Since successfully penetrating conidia on knockdown plants were inevitably arrested before extending into large mycelia, we hypothesize that TaATG6s play only a weak positive role, probably in the early phase of $B g t$ invasion, in the Pm21-triggered resistance response. Recent studies on autophagy-specific atg gene mutants support the involvement of autophagy in plant immune responses [6,8]. The Bgt-enhanced occurrence of LysoTracker Red-stained autolysosome-like structures in control plants of 92R137/Yangmai $158^{7}$ (Pm21), which "disappeared" in TaATG6 knockdown plants, also links autophagy to ATG6 during the wheat immune response to Bgt. Positive roles of autophagy in plant resistance responses have been reported $[16,17,43,44,54]$. Similar to our finding of a weak, positive role of TaATG6s in the Pm21-triggered resistance response to Bgt, Arabidopsis atg7 and atg 9 mutants are compromised in the early restriction of avirulent $P$. syringae growth, but retain the capacity to induce other defense responses [42].

Contrary to their positive roles in the Pm21-triggered resistance response, TaATG6s had negative roles in the basal resistance of the susceptible line Yangmai 158. Knocking down TaATG6s enhanced the resistance of Yangmai 158 plants to Bgt mycelial extension and sporulation. Our expression data showed that, compared with their performances in resistance plants of 92R137/Yangmai $158^{7}$ (Pm21), TaATG6s were absent for the first response to $B g t$, which occurred within 0-10 hai, but were intensely involved in the second response to Bgt, which occurred after 16 hai, in the susceptible plants of Yangmai 158 . The large quantity of successfully penetrating 
A

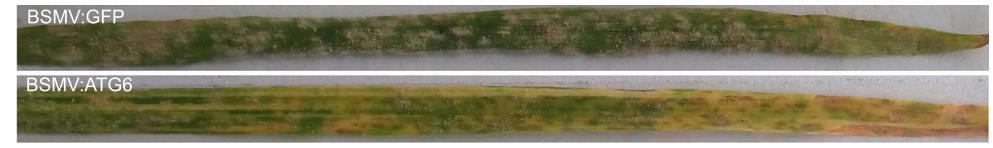

B

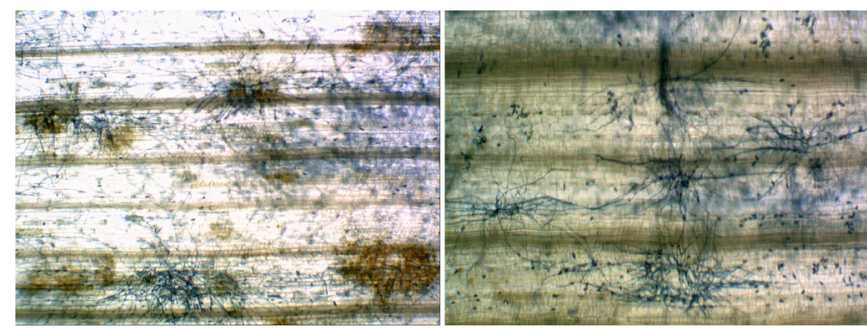

Figure 8 Lesions and chlorosis are induced by Blumeria graminis f. sp. tritici (Bgt) on wheat autophagy-related ATG6 knockdown susceptible plants.. (A) Representative images of mildew colonies, leaf lesions and chlorosis on the sixth leaves of BSMV-treated Yangmai 158 plants at 10 days after inoculation (dai) with Bgt conidia. (B) Microscopic observation of growth-retarded small mycelia associated (left panel) or not associated (right panel) with leaf cell death on BSMV:ATG6-treated Yangmai 158 plants at 10 dai.

Bgt conidia on susceptible plants may induce the highly upregulated expression of TaATG6s after 16 hai. The retarded mycelial spread and sporulation on TaATG6 knockdown susceptible plants suggested that, in wildtype susceptible plants, the ATG6 recruitment after 16 hai might be beneficial for the further development of Bgt mycelium. Thus, high levels of TaATG6s, and certain TaATG6-regulated pathways, in susceptible host cells might be hijacked by Bgt after the penetration stage to facilitate its pathogenicity, leading them to function negatively in the basal resistance of susceptible plants. The autophagy process in host cells is a potential target because of its function in the remobilization of cellular nutrients, which may be exploited by Bgt to meet the nutrient supply for fungus proliferation. Negative roles of autophagy in plant basal resistance have been reported in the susceptible responses of Arabidopsis to the powdery mildew fungus Golovinomyces cichoracearum [55] and to the bacterial pathogen P. syringae [44,54]. However, we failed to observe autophagy activity in Bgtinfected cells of wild-type Yangmai 158 plants using the LysoTracker Red staining method, probably because of the very low induction level (data not shown). Considering this and the pleiotropy of ATG6, we cannot exclude other ATG6- or PI3P-regulated pathways, or their interplay with autophagy, in underpinning the negative role of ATG6 in the basal resistance of susceptible wheat plants to powdery mildew.

Cell death was induced by growth-retarded small mycelia of Bgt on the leaves of TaATG6 knockdown plants of Yangmai 158. However, cell death does not seem to be the reason for the enhanced resistance because it occurred after the exhibition of enhanced resistance and was not induced by all small mycelia. Similarly, powdery mildew induced lesions, but they were not necessarily coupled with the enhanced resistance of susceptible Arabidopsis atg2 mutants [55]. The enhanced resistance of Arabidopsis pmr mutants to the powdery mildew pathogen Erysiphe cichoracearum and autophagy-defective mutants to $P$. syringae is also not associated with cell death $[54,56]$. Since ATG2 is autophagy-specific, the powdery mildew-induced cell death of susceptible Arabidopsis atg 2 mutants indicates a pro-survival role of autophagy in this compatible interaction [55]. However, in view of the weak Bgt-induced autophagy level and the pleiotropy of ATG6, whether autophagy alone or combined with other ATG6-regulated pathways underlies the suppression function of TaATG6s on Bgt-induced cell death of susceptible wheat plants needs to be determined.

\section{Conclusions}

In this study we provide evidence from yeast mutant complementation, subcellular localization of GFP fusions, and detection of autophagy levels in knockdown plants to show that the three identified wheat ATG6s are essential for autophagy biogenesis. Bgt-induced expression patterns, and the altered symptoms of powdery mildew on knockdown plants, suggest that TaATG6s are implicated in wheat immunity to Bgt, playing a weak, positive role in the Pm21-triggered resistance response and a negative role in the basal resistance of the susceptible line Yangmai158. These findings lay the foundations for a better understanding of autophagy mechanisms in crop plants and the role of ATG6 in wheat immunity. 


\section{Additional files}

Additional file 1: Figure S1. Sequence comparison and phylogenetic relationship of eukaryotic autophagy-related ATG6 proteins. (A) Sequence comparison between wheat ATG6 proteins and their homologs. The predicted amino acid sequences of wheat TaATG6a, $6 b$ and $6 c$ were aligned with homologous ATG6s from Arabidopsis (AtATG6, AAK62668), yeast (ScATG6, Q02948) and human (HsATG6, Q14457). The alignment was generated in ClustalX 2.1 and viewed in the GeneDoc 2.7 program. Numbers on the right indicate amino acid residue positions. Similarity was coded as follows: $100 \%$, black; 80 to $100 \%$, dark gray; 60 to $80 \%$, light gray; and $<60 \%$, white. The ATG6 domain (Pfam PF04111) region is under the straight lines. (B) Phylogenetic relationship of plant ATG6s. The phylogenetic tree was generated with MEGA 5 using the neighbor-joining method. The reliability of internal branches was assessed by bootstrapping, with 1000 bootstrap replicates, and the values are shown in percentages, with a branching cut-off at 50\%. Wheat ATG6s are indicated by diamonds. Figure S2. Diagram of the exon-intron structures of wheat ATG6 genomic ORF sequences. Exons (boxes) and introns (lines between boxes) are drawn to scale, except introns indicated by question marks, which represent there are gaps between available genomic sequences. Two vertical short lines in intron 3 of TaATG6b represent direct short sequence repeats (TAGACTTAAATCATACTCC) delimiting the non-LTR retrotransposon (RT) sequence.

Additional file 2: Table S1. List of primers used in this paper.

\section{Abbreviations}

ATG: Autophagy-related gene; BSMV: Barley stripe mosaic virus; GFP: Green fluorescent protein; PAS: Pre-autophagosomal structure/phagophore assembly site; PI3K: Phosphatidylinositol 3-kinase; VIGS: Virus-induced gene silencing.

\section{Competing interests}

The authors declare that they have no competing interests.

\section{Authors' contributions}

JY and HW designed the research. JY, HS, WZ, DP and YH conducted the experiments and analyzed the data. HW wrote the manuscript. All authors read and approved the final manuscript.

\section{Acknowledgements \\ We thank the other members of our laboratory for help with the research and for insightful remarks. This research was supported by the Natural Science Foundation of Tianjin, grants 12JCQNJC09700 and 12JCZDJC23000, and the Fok Ying-Tong Education Foundation for Young Teachers in the Higher Education Institutions of China, grant 131026.}

\section{Received: 23 November 2014 Accepted: 16 March 2015} Published online: 01 April 2015

\section{References}

1. Jones JD, Dangl JL. The plant immune system. Nature. 2006;444:323-9.

2. Chisholm ST, Coaker G, Day B, Staskawicz BJ. Host-microbe interactions: shaping the evolution of the plant immune response. Cell. 2006;124:803-14.

3. Liu Y, Bassham DC. Autophagy: pathways for self-eating in plant cells. Annu Rev Plant Biol. 2012;63:215-37.

4. Li F, Vierstra RD. Autophagy: a multifaceted intracellular system for bulk and selective recycling. Trends Plant Sci. 2012;17:526-37.

5. Klionsky DJ, Abdalla FC, Abeliovich H, Abraham RT, Acevedo-Arozena A, Adeli K, et al. Guidelines for the use and interpretation of assays for monitoring autophagy. Autophagy. 2012:8:445-544.

6. Talbot NJ, Kershaw MJ. The emerging role of autophagy in plant pathogen attack and host defence. Curr Opin Plant Biol. 2009;12:444-50.

7. Yoshimoto K, Takano Y, Sakai Y. Autophagy in plants and phytopathogens. FEBS Lett. 2010;584:1350-8.

8. Hayward AP, Dinesh-Kumar SP. What can plant autophagy do for an innate immune response? Annu Rev Phytopathol. 2011;49:557-76.

9. Zhou J, Yu JQ, Chen Z. The perplexing role of autophagy in plant innate immune responses. Mol Plant Pathol. 2014;15:637-45.
10. Teh OK, Hofius D. Membrane trafficking and autophagy in pathogen-triggered cell death and immunity. J Exp Bot. 2014;65:1297-312.

11. Kihara A, Noda T, Ishihara N, Ohsumi Y. Two distinct Vps34 30 phosphatidylinositol 3-kinase complexes function in autophagy and carboxypeptidase Y sorting in Saccharomyces cerevisiae. J Cell Biol. 2001;152:519-30.

12. Suzuki K, Kirisako T, Kamada Y, Mizushima N, Noda T, Ohsumi Y. The preautophagosomal structure organized by concerted functions of APG genes is essential for autophagosome formation. EMBO J. 2001;20:5971-81.

13. Obara K, Sekito T, Niimi K, Ohsumi Y. The Atg18-Atg2 complex is recruited to autophagic membranes via phosphatidylinositol 3-phosphate and exerts an essential function. J Biol Chem. 2008;283:23972-80.

14. Kametaka S, Okano T, Ohsumi M, Ohsumi Y. Apg14p and Apg6/Nps30p form a protein complex essential for autophagy in the yeast, Saccharomyces cerevisiae. J Biol Chem. 1998;273:22284-91.

15. Yang Z, Klionsky DJ. An overview of the molecular mechanism of autophagy. Curr Top Microbiol Immunol. 2009;335:1-32.

16. Liu Y, Schiff M, Czymmek K, Tallóczy Z, Levine B, Dinesh-Kumar SP. Autophagy regulates programmed cell death during the plant innate immune response. Cell. 2005;121:567-77.

17. Patel S, Dinesh-Kumar SP. Arabidopsis ATG6 is required to limit the pathogen-associated cell death response. Autophagy. 2008;4:20-7.

18. Fujiki Y, Yoshimoto K, Ohsumi Y. An Arabidopsis homolog of yeast ATG6/ VPS30 is essential for pollen germination. Plant Physiol. 2007;143:1132-9.

19. Doelling JH, Walker JM, Friedman EM, Thompson AR, Vierstra RD. The APG8/ 12-activating enzyme $A P G 7$ is required for proper nutrient recycling and senescence in Arabidopsis thaliana. J Biol Chem. 2002;277:33105-14.

20. Hanaoka H, Noda T, Shirano Y, Kato T, Hayashi H, Shibata D, et al. Leaf senescence and starvation-induced chlorosis are accelerated by the disruption of an Arabidopsis autophagy gene. Plant Physiol. 2002;129:1181-93.

21. Xiong Y, Contento AL, Bassham DC. AtATG18a is required for the formation of autophagosomes during nutrient stress and senescence in Arabidopsis thaliana. Plant J. 2005;42:535-46.

22. Harrison-Lowe NJ, Olsen LJ. Autophagy protein 6 (ATG6) is required for pollen germination in Arabidopsis thaliana. Autophagy. 2008;4:339-48.

23. Petiot A, Faure J, Stenmark H, Gruenberg J. PI3P signaling regulates receptor sorting but not transport in the endosomal pathway. J Cell Biol. 2003;162:971-9.

24. Xue HW, Chen X, Mei Y. Function and regulation of phospholipid signalling in plants. Biochem J. 2009:421:145-56.

25. Qin G, Ma Z, Zhang L, Xing S, Hou X, Deng J, et al. Arabidopsis AtBECLIN 1/ AtAtg6/AtVps30 is essential for pollen germination and plant development. Cell Res. 2007;17:249-63.

26. Lee Y, Kim ES, Choi Y, Hwang I, Staiger CJ, Chung YY, et al. The Arabidopsis phosphatidylinositol 3-kinase is important for pollen development. Plant Physiol. 2008;147:1886-97.

27. Xu N, Gao XQ, Zhao XY, Zhu DZ, Zhou LZ, Zhang XS. Arabidopsis AtVPS15 is essential for pollen development and germination through modulating phosphatidylinositol 3-phosphate formation. Plant Mol Biol. 2011;77:251-60.

28. Kurusu T, Koyano T, Hanamata S, Kubo T, Noguchi Y, Yagi C, et al. OsATG7 is required for autophagy-dependent lipid metabolism in rice postmeiotic anther development. Autophagy. 2014;10:1-11.

29. Liang XH, Kleeman LK, Jiang HH, Gordon G, Goldman JE, Berry G, et al. Protection against fatal Sindbis virus encephalitis by beclin, a novel Bcl-2interacting protein. J Virol. 1998;72:8586-96.

30. Livak KJ, Schmittgen TD. Analysis of relative gene expression data using real-time quantitative $P C R$ and the $2-\triangle \triangle C T$ method. Methods. 2001;25:402-8

31. Gietz RD, Schiestl RH. Quick and easy yeast transformation using the LiAc/SS carrier DNA/PEG method. Nat Protoc. 2007;2:35-7.

32. Holzberg S, Brosio P, Gross C, Pogue GP. Barley stripe mosaic virus-induced gene silencing in a monocot plant. Plant J. 2002;30:315-27.

33. Zhou H, Li S, Deng Z, Wang X, Chen T, Zhang J, et al. Molecular analysis of three new receptor-like kinase genes from hexaploid wheat and evidence for their participation in the wheat hypersensitive response to stripe rust fungus infection. Plant J. 2007:52:420-34.

34. Van Wees S: Trypan Blue Stain for Fungi, oomycetes, and dead plant cells. In Arabidopsis: a laboratory manual. Chapter 4. Edited by Weigel D, Glazebrook J. Cold Spring Harbor: Cold Spring Harbor Laboratory Press; 2002: 86-87.

35. A database for wheat genomic sequences and physical and genetic maps [http://wheat-urgi.versailles.inra.fr] 
36. Liang XH, Jackson S, Seaman M, Brown K, Kempkes B, Hibshoosh H, et al. Induction of autophagy and inhibition of tumorigenesis by beclin 1. Nature. 1999:402:672-6.

37. Obara K, Sekito T, Ohsumi Y. Assortment of phosphatidylinositol 3-kinase complexes-Atg14p directs association of complex I to the preautophagosomal structure in Saccharomyces cerevisiae. Mol Biol Cell. 2006;17:1527-39.

38. Kihara A, Kabeya Y, Ohsumi Y, Yoshimori T. Beclin-phosphatidylinositol 3-kinase complex functions at the trans-Golgi network. EMBO Rep. 2001;2:330-5.

39. Li BX, Li CY, Peng RQ, Wu XJ, Wang HY, Wan DS, et al. The expression of beclin 1 is associated with favorable prognosis in stage IIIB colon cancers. Autophagy. 2009;5:303-6.

40. Kang R, Zeh HJ, Lotze MT, Tang D. The Beclin 1 network regulates autophagy and apoptosis. Cell Death Differ. 2011;18:571-80.

41. Moriyasu Y, Hattori M, Jauh G, Rogers JC. Alpha tonoplast intrinsic protein is specifically associated with vacuole membrane involved in an autophagic process. Plant Cell Physiol. 2003:44:795-802.

42. Hofius D, Schultz-Larsen T, Joensen J, Tsitsigiannis DI, Petersen NH, Mattsson $\mathrm{O}$, et al. Autophagic components contribute to hypersensitive cell death in Arabidopsis. Cell. 2009;137:773-83.

43. Lai Z, Wang F, Zheng Z, Fan B, Chen Z. A critical role of autophagy in plant resistance to necrotrophic fungal pathogens. Plant J. 2011;66:953-68.

44. Kwon SI, Cho HJ, Kim SR, Park OK. The Rab GTPase RabG3b positively regulates autophagy and immunity-associated hypersensitive cell death in Arabidopsis. Plant Physiol. 2013;161:1722-36.

45. Inoue $Y$, Suzuki $T$, Hattori $M$, Yoshimoto $K$, Ohsumi $Y$, Moriyasu Y. AtATG genes, homologs of yeast autophagy genes, are involved in constitutive autophagy in Arabidopsis root tip cells. Plant Cell Physiol. 2006:47:1641-52.

46. Bassham DC. Plant autophagy-more than a starvation response. Curr Opin Plant Biol. 2007;10:587-93.

47. Yoshimoto K, Jikumaru Y, Kamiya Y, Kusano M, Consonni C, Panstruga R, et al. Autophagy negatively regulates cell death by controlling NPR1dependent salicylic acid signaling during senescence and the innate immune response in Arabidopsis. Plant Cell. 2009;21:2914-27.

48. Rana RM, Dong S, Ali Z, Huang J, Zhang HS. Regulation of ATG6/Beclin-1 homologs by abiotic stresses and hormones in rice (Oryza sativa L.). Genet Mol Res. 2012:11:3676-87.

49. Welters P, Takegawa K, Emr SD, Chrispeels MJ. ATVPS34, a Ptdlns 3-kinase of Arabidopsis thaliana is an essential protein with homology to a calciumdependent lipid-binding domain. Proc Natl Acad Sci U S A. 1994;91:11398-402.

50. Yoshimoto K, Hanaoka H, Sato S, Kato T, Tabata S, Noda T, et al. Processing of ATG8s, ubiquitin-like proteins, and their deconjugation by ATG4s are essential for plant autophagy. Plant Cell. 2004;16:2967-83.

51. Liu GS, Sheng XY, Greenshields DL, Ogieglo A, Kaminskyj S, Selvaraj G, et al. Profiling of wheat class III peroxidase genes derived from powdery mildewattacked epidermis reveals distinct sequence-associated expression patterns. Mol Plant Microbe Interact. 2005;18:730-41.

52. Bhuiyan NH, Selvaraj G, Wei Y, King J. Gene expression profiling and silencing reveal that monolignol biosynthesis plays a critical role in penetration defense in wheat against powdery mildew invasion. J Exp Bot. 2009;60:509-21.

53. Hückelhoven R, Kogel KH. Reactive oxygen intermediates in plant-microbe interactions: who is who in powdery mildew resistance? Planta. 2003;216:891-902.

54. Lenz HD, Haller E, Melzer E, Kober K, Wurster K, Stahl M, et al. Autophagy differentially controls plant basal immunity to biotrophic and necrotrophic pathogens. Plant J. 2011;66:818-30.

55. Wang Y, Nishimura MT, Zhao T, Tang D. ATG2, an autophagy-related protein, negatively affects powdery mildew resistance and mildew-induced cell death in Arabidopsis. Plant J. 2011;68:74-87.

56. Vogel J, Somerville S. Isolation and characterization of powdery mildewresistant Arabidopsis mutants. Proc Natl Acad Sci U S A. 2000;97:1897-902.

\section{Submit your next manuscript to BioMed Central and take full advantage of:}

- Convenient online submission

- Thorough peer review

- No space constraints or color figure charges

- Immediate publication on acceptance

- Inclusion in PubMed, CAS, Scopus and Google Scholar

- Research which is freely available for redistribution

Submit your manuscript at www.biomedcentral.com/submit 